\title{
Model for surface plasmon polariton mediated interaction between two dielectric-filled nanowaveguides
}

\section{Daniel K. Nikolov}

\section{Mary Grace M. Velasco}

\section{Huizhong Xu \\ xuh@stjohns.edu}

\begin{abstract}
Department of Physics, College of Liberal Arts and Sciences, St. Johns University, 8000 Utopia Parkway, Jamaica, NY 11439, USA

Department of Physics, College of Liberal Arts and Sciences, St. Johns University, 8000 Utopia Parkway, Jamaica, NY 11439, USA

Department of Physics, College of Liberal Arts and Sciences, St. Johns University, 8000 Utopia Parkway, Jamaica, NY 11439, USA
\end{abstract}

We propose an analytical model that describes the interaction between two apertures based on the excitation and propagation of surface plasmon polaritons. The model assumes no fitting parameters, and only requires coefficients that describe elementary processes occurring with a single aperture. We then use the model to predict both the far-field transmission and the near field intensity of two dielectric-filled cylindrical nanowaveguides separated by a varying distance. The choice of material and geometry for the nanowaveguides is based on a previous study where it was demonstrated that transmission of more than $40 \%$ can be achieved through isolated waveguides of diameter less than one tenth of the incident wavelength. In this current study, we found that both the transmission and the near field intensity of a nanowaveguide pair display an oscillatory behavior, however the oscillations in the near field intensity curve decay more rapidly. We compared the model predictions with results from three dimensional finite element simulations and the two are in excellent agreement. In addition, we analyzed the dependence of transmission on the polarization of the incident light and found that the interaction is negligible when the axis connecting the two waveguides is perpendicular to the polarization of the incident light.

[DOI: http://dx.doi.org/10.2971/jeos.2012.12044]

Keywords: Surface plasmon polaritons, nanowaveguides, coupling

\section{INTRODUCTION}

There has been a recent interest in the transmission properties of subwavelength structures since Ebbesen's discovery of extraordinary transmission through aperture arrays of subwavelength size [1]. Subsequently, many have sought to explain this phenomenon by considering the various possible mechanisms involved [2]-[10]. It is generally accepted that interference between apertures via surface plasmon polaritons (SPPs) excited at the interface between the aperturecontaining metal film and the surrounding dielectric materials plays a key role in the extraordinary transmission of aperture arrays [11].

In order to quantitatively explain the characteristic features of the extraordinary transmission spectrum, considerable research has been conducted to understand the excitation of SPPs by subwavelength nanostructures including single circular apertures [12, 13], slits and grooves [14]-[16], and the SPP enabled interactions between these structures [8]-[10],[17]-[19]. An oscillatory optical response is typically observed in coupled nanostructure systems and can be well captured by various SPP models. However, the details of the optical response vary considerably depending on the actual structure parameters, such as the film thickness [19] and the aperture size [20]. Furthermore, an analytical model that can yield quantitative agreement with experiments, with respect to the amplitudes of the transmission resonances for example, is still lacking or can only be accomplished by using fitting parameters.

In this work, we develop an analytical model to study the transmission of light through two dielectric-filled cylindrical waveguides separated by an arbitrary distance. A similar configuration with a single aperture has been previously analyzed for its promise of tight confinement with strong transmission [21, 22]. By analyzing the basic processes underlying this transmission, analytical expressions for both the far-field transmission and the near field intensity are derived. Since all coefficients or functions required to evaluate the expressions can be obtained from the analysis of a single waveguide, no fitting parameters are needed in our model. The model predictions are then verified using finite element simulations. Furthermore, we study the dependence of the transmission on the polarization of the incident light and obtain analytical results that are in very good agreement with simulations.

\section{TRANSMISSION THROUGH A SINGLE APERTURE}

The geometry used for the analysis of transmission through a single nanowaveguide consists of four components: a metal film of thickness $d$, a cylindrical aperture with height $d$ and di- 
ameter $2 r$ spanning the film, a substrate below the metal film, and an exit material above the metal film. The structure is illuminated from below the substrate layer by incident light linearly polarized in the $\mathrm{x}$-direction with wavelength $\lambda$. The relative permittivity of the metal film and the dielectric core are $\varepsilon_{\text {metal }}$ and $\varepsilon_{\text {core }}$ respectively; and that of the substrate and the exit material are $\varepsilon_{\text {sub }}$ and $\varepsilon_{\text {exit }}$ respectively. The relative permeability for all materials is assumed to be equal to 1 .

The normalized transmission $T$, which is defined as the ratio of the transmitted photon flux to the incident photon flux on the aperture area, is used to quantify the transmission properties of the aperture:

$$
T=\frac{\int \overline{P_{z}} d S}{\pi a^{2} \cdot I_{0}}
$$

where $I_{0}$ is the intensity of the incident light and $\overline{P_{z}}$ denotes the component of the time averaged Poynting vector along the $\mathrm{z}$-direction. The integration is performed over a circular area of $\pi r^{2}$ covering the aperture at its exit.

Because of the small size of the aperture, a single-mode model can be used to analyze the propagation of light inside the cylindrical waveguide [21, 23]. For a single waveguide, the squared ratio of the x-component $E_{x}$ of the electric field to the incident electric field $E_{0},\left|E_{x} / E_{0}\right|^{2}$, is given by [21]:

$$
\left|\frac{E_{x}}{E_{0}}\right|_{\text {single }}^{2}=\left|t_{1} t_{2 n} \exp \left(-k_{z}^{\prime \prime} d\right) F_{f p}\right|^{2}
$$

where $d$ is the depth of waveguide, $t_{1}$ and $t_{2 n}$ are the coupling coefficients from an incident plane wave in the substrate medium to the fundamental mode in the waveguide and that from the fundamental mode in the waveguide to the outgoing near field in the exit material respectively, $F_{f p}$ is the FabryPerot factor and $k_{z}^{\prime}$ and $k_{z}^{\prime \prime}$ refer to the real and imaginary parts of the z-direction propagation constant $k_{z}$ respectively. We can write a similar expression for the transmission $T_{\text {single }}$ by replacing the near field transmission amplitude $t_{2 n}$ with the far field equivalent $t_{2}$ :

$$
T_{\text {single }} \propto\left|t_{1} t_{2} \exp \left(-k_{z}^{\prime \prime} d\right) F_{f p}\right|^{2}
$$

\section{MODEL FOR TWO COUPLED WAVEGUIDES}

The geometry that we use in our study of two coupled waveguides is similar to the one used for a single aperture. Figure 1 shows the $x$-z-cross-sectional view of such a geometry. We analyze two identical waveguides with diameter $2 r=40 \mathrm{~nm}$ and thickness $d=100 \mathrm{~nm}$. The choice of the materials is such that it would support optimal transmission through a single aperture [22]. Hence, the apertures are filled with $\mathrm{ZnO}$, positioned in an infinitely large silver film of thickness $d=100 \mathrm{~nm}$, the exit material is water and the substrate is fused silica. The separation between the centers of the two waveguides is $\rho$ and varies from $40 \mathrm{~nm}$ (zero separation) to $1.2 \mu \mathrm{m}$. The incident light is linearly polarized in the $x$-direction and propagates in the positive z-direction. The wavelength used throughout the study

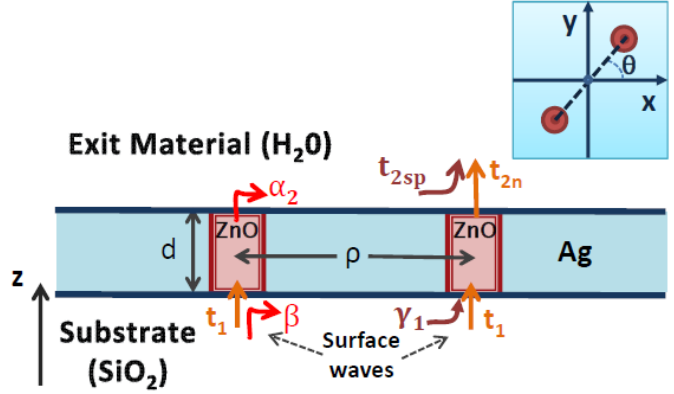

FIG. 1 The $x-z$ cross-sectional geometry of the model for two coupled nanowaveguides. The inset shows the $x-y$ cross sectional geometry.

is fixed at $488 \mathrm{~nm}$. The apertures are initially positioned parallel to the incident polarization direction. The angle $\theta$ between the axis connecting the waveguides and the polarization is then varied to be $30^{\circ}, 60^{\circ}$, and $90^{\circ}$ (see inset in Figure 1).

Figure 1 shows the possible processes taking place in the setup of two coupled nanowaveguides. First, the incident light can enter each waveguide and couple with the fundamental model propagating inside the waveguide, and $t_{1}$ is the coupling coefficient of the incident plane waves to the fundamental mode. At the same time, the incident plane waves are also scattered by the nanowaveguide to become SPPs propagating on the $\mathrm{SiO}_{2} / \mathrm{Ag}$ interface, and $\beta$ is the coefficient of scattering of the incident plane waves to the SPPs. As the SPPs reach the other waveguide, they can enter the waveguide and become the fundamental mode, and $\gamma_{1}$ is the coupling coefficient of SPPs on the $\mathrm{SiO}_{2} / \mathrm{Ag}$ interface to the fundamental mode inside the waveguide. On the $\mathrm{Ag} / \mathrm{H}_{2} \mathrm{O}$ interface, the fundamental mode can exit from each waveguide into the exit material, and $t_{2}$ and $t_{2 n}$ denote the coupling coefficients of the fundamental mode to the outgoing far field and the near field just above the aperture respectively. Also, the fundamental mode can emerge from each waveguide and become SPPs propagating on the $\mathrm{Ag} / \mathrm{H}_{2} \mathrm{O}$ interface, and $\alpha_{2}$ is the coefficient of scattering of the fundamental mode inside each waveguide to the SPPs. As SPPs reach the other waveguide, they can be scattered, and $t_{2 s p}$ is the coefficient of scattering of SPPs to the near field just above the aperture in the exit material. We note another two coefficients $\gamma_{2}$ (similar to $\gamma_{1}$ but on the exit side) and $\alpha_{1}$ (similar to $\alpha_{2}$ but on the entrance side) also exist. All the coefficients introduced here can be found by studying a single isolated waveguide.

Since the two waveguides are identical, we just need to consider the transmission through one of the waveguides, for example, the waveguide on the right side. The presence of the left waveguide will have two effects. First, the SPPs scattered from the left waveguide can enter the right waveguide as a fundamental mode after propagating for a distance of $\rho$ at the entrance metal-dielectric interface. Second, the fundamental mode can exit from the right waveguide as SPPs propagating at the exit metal-dielectric interface and enter the left waveguide as a fundamental mode moving along the negative $\mathrm{z}$ axis, and then exit from the left waveguide as SPPs propagating at the entrance metal-dielectric interface 
and reenter the right waveguide as a fundamental mode. This completes a round trip going through both waveguides. The round trip can be repeated an arbitrary number of times.

If we let $f_{1}(\rho, \theta)$ and $f_{2}(\rho, \theta)$ denote the function form of the surface waves at the entrance and exit metal-dielectric interfaces respectively, the first effect described above will provide another component of the fundamental mode in the right waveguide with a strength of $\beta \gamma_{1} f_{1}(\rho, \theta)$ but polarized along the axis connecting the two waveguides. Therefore the total strength of the fundamental mode in the right waveguide will be given by:

$$
t_{1}^{\prime}=t_{1}+\beta \gamma_{1} f_{1}(\rho, \theta) \cos \theta
$$

where the first term accounts for the contribution from the incident plane waves. The coupling of the waveguides through the large round trip can be described by a coupling factor,

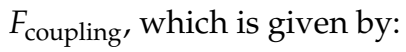

$$
F_{\text {coupling }}=\frac{1}{1-\alpha_{1} \alpha_{2} \gamma_{1} \gamma_{2} f_{1}(\rho, \theta) f_{2}(\rho, \theta) \cos ^{2} \theta F_{f p}^{2}}
$$

where the Fabry-Perot factor accounts for the fact that the fundamental mode can bounce back and forth inside each of the two waveguides before exiting as SPPs. However, the coefficients $\alpha_{1}, \alpha_{2}, \gamma_{1}, \gamma_{2}$ are all very small for the setup used in this study, resulting in a coupling factor of essentially 1.

Taking into account these two effects, we can now write down the following expression for the area normalized transmission through each of the two apertures:

$$
\begin{aligned}
T_{\text {couple }} & \propto\left|t_{1}^{\prime} t_{2} \exp \left(-k_{z}^{\prime \prime} d\right) F_{f p} F_{\text {coupling }}\right|^{2} \\
& \approx\left|t_{1}^{\prime} t_{2} \exp \left(-k_{z}^{\prime \prime} d\right) F_{f p}\right|^{2}
\end{aligned}
$$

where $t_{1}^{\prime}$ is given by Eq. (4) and $t_{2}$ is the coupling coefficient of the fundamental mode to the outgoing far filed as defined above.

To calculate the near field intensity, we need to consider the fact that SPPs originating from the left waveguide propagating at the exit metal-dielectric interface are scattered by the right waveguide into near fields with the scattering coefficient $t_{2 s p}$. Taking into consideration this field and the one exiting directly from the waveguide, we have the following expression for the resulting normalized near field intensity at the exit:

$$
\begin{aligned}
\left|\frac{E_{x}}{E_{0}}\right|_{\text {couple }}^{2} & =\left|t_{1}^{\prime} t_{2 n}^{\prime} \exp \left(-k_{z}^{\prime \prime} d\right) F_{f p} F_{\text {coupling }}\right|^{2} \\
& \approx\left|t_{1}^{\prime} t_{2 n}^{\prime} \exp \left(-k_{z}^{\prime \prime} d\right) F_{f p}\right|^{2}
\end{aligned}
$$

where the new near field coupling coefficient $t_{2 n}^{\prime}$ is given by

$$
t_{2 n}^{\prime}=t_{2 n}+\alpha_{2} t_{2 s p} f_{2}(\rho, \theta) \cos \theta
$$

and the $\cos \theta$ term is included because the additional near field from SPPs is polarized along the direction of the axis connecting the waveguides.
From Eqs. (2), (3), (6), and (7), ratios of far field transmission and near field intensity for coupled waveguides to a single waveguide can be obtained:

$$
\begin{aligned}
& \frac{T_{\text {couple }}}{T_{\text {single }}}=\left|\frac{t_{1}^{\prime}}{t_{1}}\right|^{2}=\left|1+\frac{\beta \gamma_{1}}{t_{1}} f_{1}(\rho, \theta) \cos \theta\right|^{2} \\
& \frac{\left|E_{x}\right|_{\text {couple }}^{2}}{\left|E_{x}\right|_{\text {single }}^{2}}=\left|\frac{t_{1}^{\prime}}{t_{1}} \frac{t_{2 n}^{\prime}}{t_{2 n}}\right|^{2} \\
&=\mid\left(1+\frac{\beta \gamma_{1}}{t_{1}} f_{1}(\rho, \theta) \cos \theta\right) \times \\
&\left.\left(1+\frac{\alpha_{2} t_{2 s p}}{t_{2 n}} f_{2}(\rho, \theta) \cos \theta\right)\right|^{2}
\end{aligned}
$$

Next we need to know the actual form of $f_{1}(\rho, \theta)$ and $f_{2}(\rho, \theta)$ and the values of the coefficients before evaluating Eqs. (9) and (10). It is well known that the SPPs at the interface of a nonmagnetic metal and a dielectric can only be transverse magnetic (TM) waves with the propagating constant described by [24]

$$
k_{s p}=k_{0} \sqrt{\frac{\varepsilon_{\text {metal }} \varepsilon_{\text {dielectric }}}{\varepsilon_{\text {metal }}+\varepsilon_{\text {dielectric }}}}
$$

where $\varepsilon_{\text {metal }}$ and $\varepsilon_{\text {dielectric }}$ are the relative permittivity of the metal and the dielectric respectively. Also, as shown in a previous study [13], the radial dependence of the $E_{z}$ field scattered by a circular aperture can be well described by the first kind Hankel function $H_{n}^{(1)}\left(k_{s p} \rho\right)$. Thus, after taking into account the angular dependence of the TM waves, we expect the propagation functions $f_{1}(\rho, \theta)$ and $f_{2}(\rho, \theta)$ to be given by:

$$
\begin{aligned}
f_{j}(\rho, \theta) & =H_{1}^{(1)}\left(k_{s p_{j}} \rho\right) \cos \theta \\
& \approx \sqrt{\frac{2}{\pi k_{s p_{j}} \rho}} \exp \left(i\left(k_{s p_{j} \rho}-\frac{3}{4} \pi\right)\right) \cos \theta
\end{aligned}
$$

where $j=1$ and 2, and the approximation holds for $k_{s p} \rho \gg 1$. The two propagating constants at the entrance and exit metaldielectric interfaces are:

$$
\begin{aligned}
& k_{s p_{1}}=k_{0} \sqrt{\frac{\varepsilon_{\mathrm{Ag}} \varepsilon_{\text {sub }}}{\varepsilon_{\mathrm{Ag}}+\varepsilon_{\text {sub }}}} \text {, and } \\
& k_{s p_{2}}=k_{0} \sqrt{\frac{\varepsilon_{\mathrm{Ag}} \varepsilon_{\text {exit }}}{\varepsilon_{\mathrm{Ag}}+\varepsilon_{\text {exit }}}}
\end{aligned}
$$

To verify that Eq. (12) describes the fields of the surface waves, we have used the three-dimensional finite element method [25] to simulate the scattering of the incident plane waves by a single $\mathrm{ZnO}$ cylinder embedded in a semi-infinite silver metal. The geometry of the single waveguide setup is shown in the inset of Figure 2 and has also been studied previously [22]. Perfectly matched layer (PML) boundary conditions [26] are assumed to terminate a computational domain of three wavelengths in the $x$ and $y$-directions and two wavelengths in the $\mathrm{z}$-directions (the thickness of the PML is $0.2 \lambda$ in all directions). The incident light consists of plane waves launched from the bottom PML. 


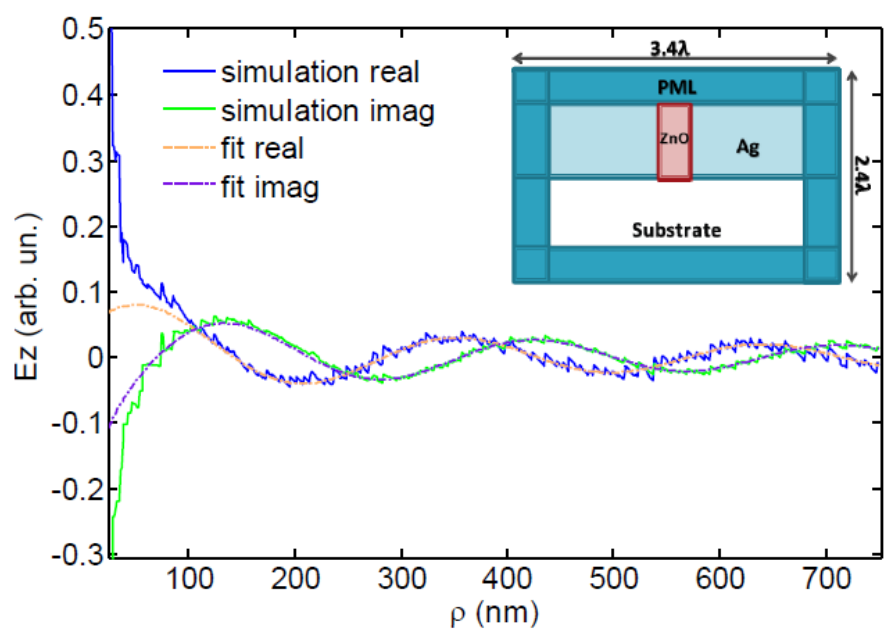

FIG. 2 The z-component of the electric field versus the distance from the source of the surface waves. The solid lines are the real and imaginary part of $E_{z}$ according to the finite element method simulation. The dashed lines are the real and imaginary part of $E_{z}$ as predicted by Eq. (14). The inset shows the $x-z$ cross sectional geometry used to calculate $\beta, t_{1}$ and $\gamma_{1}$ in the finite element method simulation.

Figure 2 shows the real and imaginary parts of the simulated $E_{z}$ field (solid lines) as a function of the radius along the $30^{\circ}$ radial direction. The dashed lines are a fit to the simulation results using the form

$$
\begin{aligned}
E_{z} & =\frac{-k_{s p 1}}{\sqrt{\varepsilon_{s u b} k_{0}^{2}-k_{s p 1}^{2}}} E_{x} \\
& =\frac{-k_{s p 1}}{\sqrt{\varepsilon_{s u b} k_{0}^{2}-k_{s p 1}^{2}}} \beta f_{1}(\rho, \theta)
\end{aligned}
$$

where $E_{x}$ is the x-component of the surface wave, and the fitting constant $\beta$ is the scattering coefficient of the incident plane waves to the SPPs and is found to be $-0.3441-0.3630 \mathrm{i}$. We can see the fits are very good for $\rho>100 \mathrm{~nm}$, indicating the validity of Eq. (12) for describing the SPPs. In addition, we confirmed that the same constant can be used to fit the $E_{z}$ field for all other radial directions. We note that the fits do not work for $\rho<40 \mathrm{~nm}$ as expected since the surface waves only exist outside the cylinder and fields inside the cylinder are described by Bessel functions.

Finally, we note that the finite element simulation setup shown in the inset in Figure 2 can also be used to evaluate the coefficients $t_{1}$ and $\gamma_{1}$. To find $\gamma_{1}$, SPPs are launched from the PML on the left side, and the $E_{x}$ field inside the waveguide is compared to the fundamental mode. To determine the values of $\alpha_{2}, t_{2 s p}$ and $t_{2 n}$, we use a similar model with the exit medium layer sitting on top of a semi-infinite waveguide embedded inside the silver metal. For $\alpha_{2}$ and $t_{2 n}$, a fundamental mode is launched from the bottom PML, and for $t_{2 s p}$ SPPs are launched from the PML on the left side. Details of the method used for calculating the coupling coefficients are described in the Appendix.

\section{RESULTS AND DISCUSSION}

Having obtained all the required values and functions to evaluate Eqs. (9) and (10), we can now predict how the near
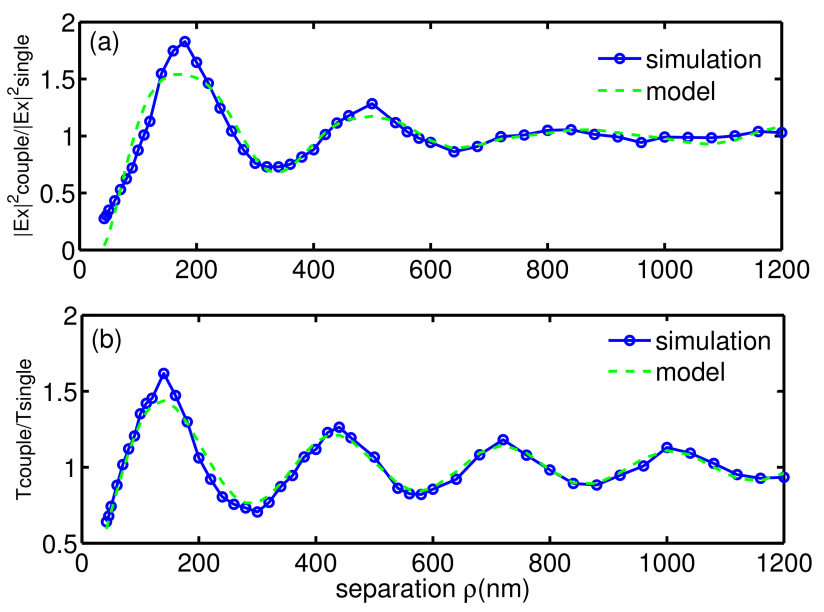

FIG. 3 The $\left|E_{x}\right|_{\text {couple }}^{2} /\left|E_{x}\right|_{\text {single }}^{2}$ ratio versus the separation between the waveguides. The circles are the ratio according to the finite element method simulation and the dashed line is the ratio predicted by Eq. (10). (b) The $T_{\text {couple }} / T_{\text {single }}$ ratio versus the separation between the waveguides. The circles are the ratio according to the finite element method simulation and the dashed line is the ratio predicted by Eq. (9).

field intensity and the far-field transmission from the two coupled waveguides behave. In Figure 3 we have plotted the calculated near field intensity and far field transmission ratios as a function of the separation distance between the two waveguides for $\theta=0^{\circ}$, i.e. when the axis connecting the waveguides is parallel to the polarization direction of the incident light. Also shown in Figure 3 are results from finite element simulations of two coupled waveguides. The simulation is done by enclosing the setup shown in Figure 1 inside a computation domain of three wavelengths in the $x$ and $y$ directions and one wavelength in the $z$ direction and terminating it with PML boundary conditions.

It can be seen that the agreement is very good for both the near field intensity and the far field transmission. We see that both the near-field intensity and the transmission modulate as a function of the separation distance. The oscillation amplitude for both curves starts at more than $50 \%$ when the waveguides are close and decreases gradually with increasing distance as a result of the attenuating amplitude of the SPPs. However, the oscillation in the near field intensity decays more rapidly than the transmission curve. When the distance between the two waveguides is greater than $1000 \mathrm{~nm}$, which corresponds to a $k_{s p 1} \rho$ of about 20 , the interaction between the two waveguides is almost negligible. Hence, at a large enough separation, the transmission through each of the two waveguides is just equal to the transmission though a single isolated waveguide. The period of both curves is about $300 \mathrm{~nm}$ which is close to the wavelength of SPPs, $2 \pi / k_{s p 1}$. However, the peak locations in the near field intensity curve are clearly shifted to the right in comparison to those in the far field transmission curve. The faster decay of the oscillation amplitude and the shift of peak locations are due to the $t_{2 s p}$ term in the expression for the near field intensity ratio, which is not present in the transmission expression. Since the fields resulting from the scattering of SPPs by an aperture are of mainly near field nature, the scattered fields only alter the near field just above the exits but do not contribute to the far 


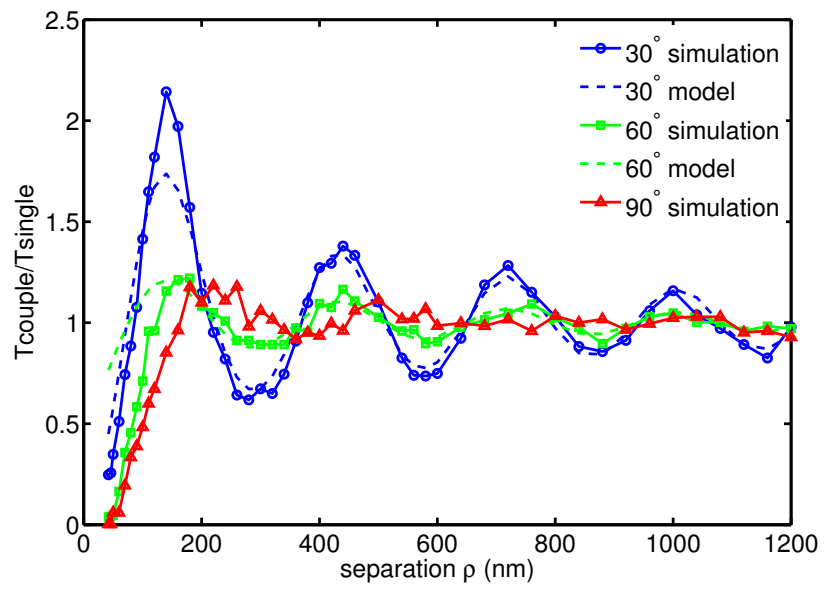

FIG. 4 The $T_{\text {couple }} / T_{\text {single }}$ ratio versus the separation between the waveguides for different angle $\theta$. The circles, squares, and triangles denote results from the finite element simulation for $\theta=30^{\circ}, 60^{\circ}$ and $90^{\circ}$. The dashed lines show the ratio predicted by Eq. (15).

field transmission.

We also calculated the transmission ratio for waveguides positioned such that the axis connecting the two waveguides forms an angle of $30^{\circ}, 60^{\circ}$ and $90^{\circ}$ with respect to the polarization of the incident light. Using the function form of $f_{1}(\rho, \theta)$, Eq. (9) can be rewritten as

$$
\frac{T_{\text {couple }}}{T_{\text {single }}}=\left|1+\frac{\beta \gamma_{1}}{t_{1}} H_{1}^{(1)}\left(k_{s p_{1}} \rho\right) \cos ^{2} \theta\right|^{2}
$$

where the $\cos ^{2} \theta$ term comes from the angular dependence of the SPPs as well as the fact that the fundamental mode produced by SPPs is polarized along the direction of the axis connecting the two waveguides. The results calculated using Eq. (15) are shown in Figure 4 for $\theta=30^{\circ}, 60^{\circ}$, and $90^{\circ}$. We note that the same set of coefficients obtained from the study of a single waveguide is used for all the analytical results shown in Figures 3 and 4. Also shown in Figure 4 are results from finite element simulations. We can see the analytical and the simulation results agree very well. The oscillation amplitude decreases gradually with increasing separation for all angles, and is quite small as the angle approaches $\pi / 2$, which is consistent with the $\cos ^{2} \theta$ dependence. This is expected, since the dominant waves propagating along the direction perpendicular to the polarization direction of the incident plane waves are TE waves, which cannot support SPPs.

The consistency between the theoretical results and the numerical data indicates that the model provides an accurate representation of the major physical phenomena behind the coupling of a pair of waveguides. In addition to revealing the general physics involved in a two-waveguide system, the model also has several other advantages. Because of its simplicity, the model can be used to calculate the transmission through two waveguides of arbitrary separation and orientation. It will also allow us to describe systems with an arbitrary choice of materials for the core, cladding, substrate and exit medium once the coupling coefficients are calculated from a single waveguide. Finally, since the model accurately describes interaction between two waveguides of arbitrary separation and orientation, it can be extended to study multiple apertures at various separations and arrangements.

\section{CONCLUSION}

We have developed an analytical model to describe the interaction between two coupled dielectric-filled nanowaveguides in a metal film. The interaction is mediated by SPPs propagating at the metal-dielectric interfaces. Analytical expressions for the near field intensity and far field transmission of coupled waveguides are obtained and only contain the propagation functions of SPPs and coefficients associated with single waveguide processes. The analytical results are compared with results from finite element simulations, and the two are in very good agreement. We have found that both the far field transmission and the near field intensity modulate with the distance between the waveguides. The oscillation amplitude decreases with increasing separation and the period is determined by the wavelength of the SPPs at the entrance metaldielectric interface. In addition, the near field intensity and far field transmission also depend on the angle between the axis connecting the waveguides and the polarization direction of the incident light. Our results have revealed that coupling between two waveguides decreases rapidly as the angle increases. In particular, the surface plasmon mediated interaction is negligible when the waveguides are placed perpendicular to the polarization direction of the incident plane waves. Because of its simplicity, we expect this model may also be useful in studying systems involving more than two waveguides in an arbitrary configuration.

\section{ACKNOWLEDGEMENTS}

This research was supported by the Seed Grant Program of St. John's University and the National Science Foundation under grant No. CBET-0953645. D.N. acknowledges the support from the Graduate Admissions Assistance Program of St. Johns University.

\section{APPENDIX: METHOD OF OBTAINING SCATTERING COEFFICIENTS}

The various coefficients in our model can be obtained from the study of a single waveguide. In Section 3, we have described the method of obtaining $\beta$, the scattering coefficient of the incident plane waves to the SPPs. The process involves two steps: the scattered surface waves of incident plane waves by a single waveguide are first obtained from finite element simulations, the coefficient is then obtained from comparing the simulated field to the expected format. Similar approaches are used to extract the other coefficients. To evaluate the coupling efficient $t_{1}$ of plane waves to the waveguide fundamental mode, a plane wave is launched from the bottom PML below the substrate (see Figure A1(a)) in our finite element simulations and the simulated $E_{x}$ field inside the semi-infinite 


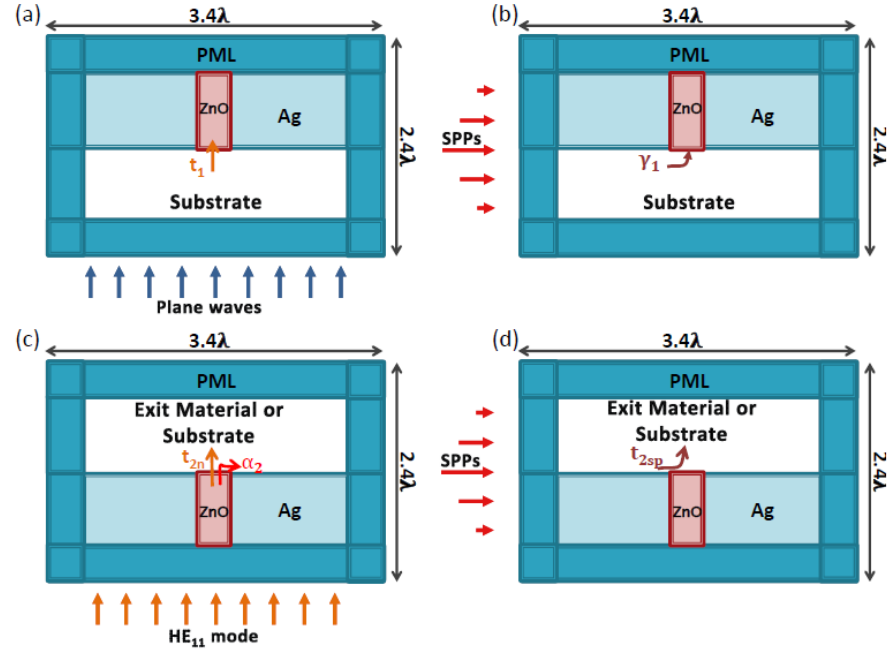

FIG. A1 The following setups are used to calculate the coupling and scattering coefficients. (a) A semi-infinite waveguide positioned above the substrate material with incident illumination of plane waves launched from the bottom PML is used for calculating the coupling coefficient $t_{1}$. (b) A semi-infinite waveguide positioned above the substrate material with incident illumination of SPPs launched from the left side PML is used for calculating the coupling coefficient $\gamma_{1}$. (c) A semi-infinite waveguide positioned below the substrate/exit material with incident illumination of the fundamental mode launched from the bottom PML is used for calculating the scattering coefficient $\alpha_{2}$ and the coupling coefficient $t_{2 n}$. (d) A semi-infinite waveguide positioned below the substrate/exit material with incident illumination of SPPs launched from the left side PML is used for calculating the coupling coefficient $t_{2 s p}$.

waveguide is then fitted with the functional form of the fundamental mode: $E_{x} \propto t_{1} \exp \left(i k_{z} z\right)$, where $k_{z}$ is the z-direction propagation constant of the waveguide fundamental mode. We note that the plane waves are launched with strength such that the $E_{x}$ component of the incident field at the center of computational domain would be unity when the entire domain is just the substrate. To evaluate the coupling coefficient $\gamma_{1}$ of SPPs to the fundamental mode, the same setup is used but with illumination of SPPs from the left side PML (see Figure A1(b)). The SPPs are launched with strength such that the $E_{x}$ component of the incident field at the center of the computation domain would be unity when the waveguide is absent. The simulated $E_{x}$ field inside the semi-infinite waveguide is then fitted with the form of the fundamental model: $E_{x} \propto \gamma_{1} \exp \left(i k_{z} z\right)$.

For the calculation of the rest of the coupling coefficients, we use a setup in which the semi-infinite waveguide is positioned below the exit material. To evaluate $\alpha_{2}$ and $t_{2 n}$, we launch fundamental mode from the bottom PML into the waveguide, and examine the fields coming out of the waveguide (see Figure A1(c)). The input fundamental mode is launched with strength such that the electric field at the center of the computational domain would be unity when the exit material is absent and the waveguide is infinite. Similar to obtaining $\beta$, $\alpha_{2}$ is then obtained by fitting the simulated $E_{z}$ field of the surface waves to the function form:

$$
\begin{aligned}
E_{z} & =\frac{-k_{s p 2}}{\sqrt{\varepsilon_{\mathrm{exit}} k_{0}^{2}-k_{s p 2}^{2}}} E_{x} \\
& =\frac{-k_{s p 2}}{\sqrt{\varepsilon_{\mathrm{exit}} k_{0}^{2}-k_{s p 2}^{2}}} \alpha_{2} f_{2}(\rho, \theta) .
\end{aligned}
$$

Next, $t_{2 n}$ is equal to the electric field just above the exit of the waveguide as shown in Figure A1(c). Finally, to calculate $t_{2 s p}$ we launch SPPs from the left side PML layer, and $t_{2 s p}$ will be equal to the electric field just above the exit of the waveguide (see Figure A1(d)). We note that the SPPs are launched with strength such that the strength of the electric field would be unity at the center of the computational domain when the waveguide is absent.

\section{References}

[1] T. W. Ebbesen, H. J. Lezec, H. F. Chaemil, T. Thio, and P. A. Wolff, "Extraordinary optical transmission through sub-wavelength hole arrays," Nature 391, 667-669 (1998).

[2] L. Martín-Moreno, F. J. García-Vidal, H. J. Lezec, K. M. Pellerin, T. Thio, J. B. Pendry, and T. W. Ebbesen, "Theory of Extraordinary Optical Transmission through Subwavelength Hole Arrays," Phys. Rev. Lett. 86, 1114-1117 (2001).

[3] W. L. Barnes, W. A. Murray, J. Dintlinger, E. Devaux, and T. W. Ebbesen, "Surface plasmon polaritons and their role in the enhanced transmission of light through periodic arrays of subwavelength holes in a metal film," Phys. Rev. Lett. 92, 107401 (1-4) (2004).

[4] H. Lezec, and T. Thio, "Diffracted evanescent wave model for enhanced and suppressed optical transmission through subwavelength hole arrays", Opt. Express 12, 3629-3651 (2004).

[5] P. Lalanne, and J. P. Hugonin, "Interaction between optical nanoobjects at metallo-dielectric interfaces," Nat. Phys. 2, 551-556 (2006).

[6] G. Gay, 0. Alloschery, B. Viaris de Lesegno, C. O’Dwyer, J. Weiner, and $\mathrm{H}$. J. Lezec, "The optical response of nanostructured surfaces and the composite diffracted evanescent wave model," Nat. Phys. 2, 262-267 (2006).

[7] F. J. Garcia de Abajo, "Colloquium: Light scattering by particle and hole arrays," Rev. Mod. Phys. 79, 1267-1290 (2007).

[8] Y. Alaverdyan, B. Sepúlveda, L. Eurenius, E. Olsson, and M. Käll, "Optical antennas based on coupled nanoholes in thin metal films," Nat. Phys. 3, 884-889 (2007).

[9] D. Pacifici, H. J. Lezec, L. A. Sweatlock, R. J. Walters, and H. A. Atwater, "Universal optical transmission features in periodic and quasiperiodic hole arrays," Opt. Express 16, 9222-9238 (2008).

[10] H. Liu, and P. Lalanne, "Microscopic theory of the extraordinary optical transmission," Nature 452, 728-731 (2008).

[11] F. J. García-Vidal, L. Martín-Moreno, T. W. Ebbesen, and L. Kuipers, "Light passing through subwavelength apertures," Rev. Mod. Phys. 82, 729-787 (2010).

[12] L. Martín-Moreno, F. J. García-Vidal, H. J. Lezec, A. Degiron, and T. W. Ebbesen, "Focusing light with a single subwavelength aperture flanked by surface corrugations," Appl. Phys. Lett. 83, 4500-4502 (2003).

[13] E. Popov, M. Nevière, A.-L. Fehrembach, and N. Bonod, "Optimization of plasmon excitation at structured apertures," Appl. 0pt. 44, 6141-6154 (2005).

[14] P. Lalanne, J. P. Hugonin, and J. C. Rodier, "Theory of Surface Plasmon Generation at Nanoslit Apertures," Phys. Rev. Lett. 95, 263902 (1-10) (2005).

[15] M. Kuttge, F. J. García de Abajo, and A. Polman, "How grooves reflect and confine surface plasmon polaritons," Opt. Express 17, 
10385-10392 (2009).

[16] S. B. Raghunathan, C. H. Gan, T. van Dijk, B. Ea Kim, H. F. Schouten, W. Ubachs, P. Lalanne, and T. D. Visser, "Plasmon switching: Observation of dynamic surface plasmon steering by selective mode excitation in a sub-wavelength slit," Opt. Express 20, 15326 (2012).

[17] H. F. Schouten, N. Kuzmin, G. Dubois, T. D. Visser, G. Gbur, P. F. A. Alkemade, H. Blok, G. W. 't Hooft, D. Lenstra, and E. R. Eliel, "Plasmon-assisted two-slit transmission: Young's experiment revisited," Phys. Rev. Lett. 94, 053901 (1-4) (2005).

[18] L. Chen, J. T. Robinson, and M. Lipson, "Role of radiation and surface plasmon polaritons in the optical interactions between a nano-slit and a nano-groove on a metal surface," Opt. Express 14, 12629-12636 (2006).

[19] J. Alegret, P. Johansson, and M. Käll, “Green's tensor calculations of plasmon resonances of single holes and hole pairs in thin gold films," New J. Phys. 10, 10544 (1-13) (2008).

[20] F. de León-Pérez, F. J. García-Vidal, and L. Martín-Moreno, "Role of surface plasmon polaritons in the optical response of a hole pair," Phys. Rev. B 84, 125414 (1-9) (2011).
[21] H. Xu, P. Zhu, H. G. Craighead, and W. W. Webb, "Resonantly enhanced transmission of light through subwavelength apertures with dielectric filling," Opt. Commun. 282, 1467-1471 (2009).

[22] M. G. Velasco, P. Cassidy, and H. Xu, "Extraordinary transmission of evanescent modes through a dielectric-filled nanowaveguide," Opt. Commun. 284, 4805-4809 (2011).

[23] L. Novotny, and C. Hafner, "Light propagation in a cylindrical waveguide with a complex, metallic, dielectric function," Phys. Rev. E 50, 4094-4106 (1994).

[24] H. Raether, Surface Plasmons on Smooth and Rough Surface and on Gratings, (Springer-Verlag, Berlin, 1988).

[25] Comsol Multyphisics RF Module, www.comsol.com

[26] J. Jin, The Finite Element Method in Electromagnetics, (2nd Edition, Wiley, New York, 2002). 